\title{
Counterterm resummation for 2PI-approximation in constant background
}

\author{
A. Patkós ${ }^{1 a, b}$ \\ ${ }^{a}$ Department of Atomic Physics, Eötvös University \\ ${ }^{b}$ Research Group for Statistical and Biological Physics \\ of the Hungarian Academy of Sciences \\ H-1117 Budapest, Hungary \\ Zs. Szép ${ }^{2}$ \\ Research Institute for Solid State Physics and Optics of the \\ Hungarian Academy of Sciences, H-1525 Budapest, Hungary
}

\begin{abstract}
Explicit counterterm construction is presented in a symmetry breaking background for the 2PI effective action of the self-interacting one-component scalar field including skeleton diagrams to $\mathcal{O}\left(\lambda^{2}\right)$. The applied strategy is an extension of our treatment of the 2PI-Hartree approximation [24]. The procedure is also applied to the $O(N)$ model.
\end{abstract}

\section{Introduction}

Renormalisation invariance is a powerful concept in constructing field theoretical models describing phenomena in a restricted range of momenta. It imposes insensitivity requirement for the physical predictions to the choice of the maximal momentum if the range characterising the phenomenon under investigation is much below that value. Renormalisable models represent a specific subclass where the maximal momentum could be sent to infinity. In view of triviality of scalar field theories, renormalisability just ensures that high precision predictions calculated with finite (but large) cut-off in such models will not depend sensitively on the cut-off.

Renormalisability criteria for perturbatively computed physical quantities as power series of the renormalised couplings represent a classical piece of knowledge [1]. One constructs at every order also the corresponding approximation of the counterterm Lagrangian, which cancels the cut-off dependence of some loop integrals. The renormalisation of partially resummed perturbative series is nontrivial because in association with the selected subset of the perturbative diagrams one has to perform also the resummation of appropriately chosen counterterm diagrams.

In the past 6-7 years considerable progress has been achieved in constructing renormalisable twoparticle-irreducible (2PI) approximate solutions of perturbatively renormalisable theories. The 2PI

E-mail: ${ }^{1}$ patkos@ludens.elte.hu, ${ }^{2}$ szepzs@achilles.elte.hu 
approximation was developed into a valuable non-perturbative tool in studies of collective features of quantum field theories both in and out of equilibrium. With its help one can investigate the evolution of the spatial distribution of ground state field condensates as well as propagators of their quasi-particle excitations.

In a series of papers [2, 3, 4] van Hees and Knoll proved renormalisability of the 2PI approximate solutions in the real time formulation of quantum field theories with help of the subtraction procedure designed by Bogoliubov, Parasiuk, Hepp, and Zimmermann (BPHZ). Their study focused on the symmetric phase with no condensate in the ground state. This line was continued in 2003-04 by Blaizot, Iancu and Reinosa [5, 6] having demonstrated iteratively that self-consistently solved propagator equations resum infinite subsets of different orders of the perturbation theory. Mainly based on diagrammatic analysis of the iterations they also constructed the counterterms necessary to balance the cut-off dependence of the loop integrals appearing in the self-consistent equations. Moreover they pointed out the importance of the large momentum asymptotics of the propagator for the determination of the cut-off dependence of the counter couplings. Related investigations were done also using the auxiliary field formulation of $\Phi^{4}$ theories (e.g. after Hubbard-Stratonovich transformation) [7, 8, 9] and also concentrating on the problem of gauge fixing parameter dependence of 2PI approximation in gauge theories [10, 11].

A complete description of how to renormalise arbitrary $n$-point functions derived in 2PI-formalism was presented by Berges, Borsányi, Reinosa and Serreau in Ref. [12], also for non-vanishing expectation value of the scalar field. The detailed analysis of that paper was mainly based also on diagrammatics and explicit counterterm formulae were displayed only partially. Since then several numerical implementations were reported for scalar theories [13, 14, 15] and also for QED [16, 17]. The symmetry aspects of the 2PI-formalism was investigated in [4. It was shown that only the so-called "external N-point functions", i.e. derivatives of the effective action with respect to the mean field fulfill Ward identities. In particular, the self-consistent propagator differs from the external two-point function and in consequence it violates Goldstone's theorem. The validity of Ward identities was discussed recently in the context of QED in [18].

Investigations of the renormalisation were performed also for a related resummation scheme using local (momentum-independent) insertions, the so-called two-particle-point-irreducible (2PPI) approximations. The renormalisation of this scheme was proved using counterterms [19] and the scheme was used in numerical works both in equilibrium [20] and out of equilibrium [21, 22. Recently in Ref. [23] a renormalisation group inspired approach was developed to investigate the connection between momentum-dependent resummation and renormalisation. There a comparison of the method with renormalisation methods relying on Bethe-Salpeter-like equations was also given.

The purpose of the present paper is to deduce the resummed counterterms in a transparent way, and provide explicit formulae as far as possible for the counter couplings. In a previous paper [24] we have shown for the 2PI-Hartree approximation that results of the iterative renormalisation of Ref. [6] can be obtained also with help of a one-step substitution. In this paper we demonstrate first the applicability of our procedure to all cases where the large momentum asymptotics of the propagator remains the same as it was in the tree level approximation. In particular, we find that in such cases there is no need to use any auxiliary Bethe-Salpeter-like equation for the determination of the 4-point counter-couplings. This feature can be studied neatly on the example of the complete two-loop skeleton diagram set of the 2PI effective action in a constant, homogeneous symmetry breaking background. The basic definitions and notations of the two models considered in this paper are introduced in Section 2. In Section 3 the construction is performed first for the case of the 
one-component real field. It is applied to the more involved case of the $O(N)$ model in Section 5 . The presentation will be detailed and still more compact than was the case of previous analyses.

The generalisation of our procedure to the case of the modified large momentum behaviour of the propagator is discussed for the one-component scalar field in Section 4. It leads to a new important intermediate step in designing the algorithm of the counterterm determination. Namely, the separation of the divergent pieces of different skeleton diagrams has to be based now on an auxiliary propagator whose asymptotics coincides with the exact large momentum behaviour and is independent of the possibly environment dependent infrared data (masses, condensates) of the theory [6]. The exact propagator is expanded around this propagator, which eventually determines the counterterms. This analysis becomes unavoidable when also the so-called "basket-ball" diagram is added to the set of skeleton diagrams determining the 2PI effective action. It is in Section 6 where we extend the analysis to the $O(N)$ model. A central question of these sections will be, how the form of subdivergences changes, whether all subdivergences can be expressed as products of a unique (possibly environment dependent) function and some explicit cut-off dependent factors, or one has to consider several independent "mixed" products. It turns out that the relevant contribution from the "basket-ball" diagram can be decomposed into the sum of terms from which some are proportional to powers of the background field, some to the finite part of the tadpole integral, therefore no new type of subdivergence shows up. This decomposition is determined by an integral kernel solving a Bethe-Salpeter type equation, which introduces quite naturally a resummation for certain 4-point functions.

As a consequence, the classification of the divergent pieces remains unchanged relative to the classes found already in the 2PI-Hartree approximation [24]: one finds overall divergences proportional to the zeroth and second power of the background field and subdivergences proportional to the finite part of the independent tadpole integrals. Their coefficients are required to vanish separately. In general, the number of renormalisation conditions arising from this requirement is larger than the number of independent counter couplings. Renormalisability of the 2PI approximation means beyond the determination of all resummed counter couplings also the demonstration that these supplementary equations do not contradict those which are used for extracting the counterterms.

The summary of our findings is given in Section 7 where we shortly outline possible approaches to renormalised (cut-off independent) numerical calculations. Some technical details relevant to the discussion presented in the main text are given in two Appendices.

\section{The models and some notations}

The complete static $\mathcal{O}\left(\lambda^{2}\right)$ skeleton 2PI functional in the broken phase of the real one-component $\Phi^{4}$ model, characterised by the vacuum expectation value $\Phi=v$ is of the following form [13]:

$$
\begin{aligned}
V[v, G] & =\frac{1}{2} m^{2} v^{2}+\frac{\lambda}{24} v^{4}-\frac{i}{2} \int_{p}\left[\ln G^{-1}(p)+D^{-1}(p) G(p)\right]+\frac{\lambda}{8}\left(\int_{p} G(p)\right)^{2} \\
& -\frac{i \lambda^{2}}{12} v^{2} \int_{k} \int_{p} G(p) G(k) G(p-k)-\frac{i \lambda^{2}}{48} \int_{k} \int_{p} \int_{q} G(q) G(p) G(k) G(p+k+q)+V_{c t}[v, G],(1)
\end{aligned}
$$


where $i D^{-1}(p)=p^{2}-m^{2}-\lambda v^{2} / 2$ is the tree-level inverse propagator. The counterterm functional is given by

$$
V_{c t}[v, G]=\frac{1}{2} \delta m_{0}^{2} v^{2}+\frac{\delta \lambda_{4}}{24} v^{4}+\frac{1}{2}\left(\delta m_{2}^{2}+\frac{\delta \lambda_{2}}{2} v^{2}\right) \int_{p} G(p)-\frac{\delta Z}{2} \int_{p} p^{2} G(p)+\frac{\delta \lambda_{0}}{8}\left(\int_{p} G(p)\right)^{2} .
$$

Note, that there is no counterterm diagram involving the "setting-sun" or the "basket-ball" diagrams. Such diagrams would be necessary only when going beyond the present level of the 2PI truncation, as will become clear in Section 4.

The generalisation of the effective action to the $N$-component scalar field with $O(N)$ symmetry in the symmetry breaking background is expressed with the following representation of the tree-level and exact propagators:

$$
i D_{a b}^{-1}(k)=\left[k^{2}-m^{2}-\frac{\lambda v_{c}^{2}}{6 N}\right]\left(P_{a b}^{\sigma}+P_{a b}^{\pi}\right)-\frac{\lambda v_{c}^{2}}{3 N} P_{a b}^{\sigma}, \quad G_{a b}(k)=G_{\sigma}(k) P_{a b}^{\sigma}+G_{\pi}(k) P_{a b}^{\pi} .
$$

Here $P_{a b}^{\sigma}=\hat{v}_{a} \hat{v}_{b}, P_{a b}^{\pi}=\delta_{a b}-\hat{v}_{a} \hat{v}_{b}$ are the relevant orthogonal projectors. $\hat{v}^{a}, a=1 \ldots N$ is the component of the unit vector giving the orientation of the symmetry breaking constant background.

In terms of the two propagating modes the 2PI-action has the form:

$$
\begin{aligned}
V\left[v_{a}, G_{\sigma}, G_{\pi}\right] & =\frac{1}{2} m^{2} v_{a}^{2}+\frac{\lambda}{24 N}\left(v_{a}^{2}\right)^{2} \\
& -\frac{i}{2} \int_{k}\left[(N-1) \ln G_{\pi}^{-1}(k)+\ln G_{\sigma}^{-1}(k)+(N-1) D_{\pi}^{-1}(k) G_{\pi}(k)+D_{\sigma}^{-1}(k) G_{\sigma}(k)\right] \\
& +\frac{\lambda}{24 N}\left[3\left(\int_{k} G_{\sigma}(k)\right)^{2}+\left(N^{2}-1\right)\left(\int_{k} G_{\pi}(k)\right)^{2}+2(N-1) \int_{k} G_{\pi}(k) \int_{p} G_{\sigma}(p)\right] \\
& -\frac{i \lambda^{2}}{36 N^{2}} v_{a}^{2} \int_{k} \int_{p}\left[3 G_{\sigma}(k) G_{\sigma}(p)+(N-1) G_{\pi}(k) G_{\pi}(p)\right] G_{\sigma}(p+k) \\
& -\frac{i \lambda^{2}}{144 N^{2}} \int_{k} \int_{p} \int_{q}\left[(N-1)\left((N+1) G_{\pi}(k) G_{\pi}(p)+2 G_{\sigma}(k) G_{\sigma}(p)\right) G_{\pi}(q) G_{\pi}(k+p+q)\right. \\
& \left.+3 G_{\sigma}(k) G_{\sigma}(p) G_{\sigma}(q) G_{\sigma}(k+p+q)\right]+V_{c t},
\end{aligned}
$$

where $i D_{\pi}^{-1}(p)=p^{2}-m^{2}-\lambda v_{a}^{2} /(6 N), i D_{\sigma}^{-1}=p^{2}-m^{2}-\lambda v_{a}^{2} /(2 N)$ are the tree-level pion and sigma inverse propagators. In this case the counterterm functional reads

$$
\begin{aligned}
V_{c t} & =\frac{1}{2} \delta m_{0}^{2} v_{a}^{2}+\frac{\delta \lambda_{4}}{24 N}\left(v_{a}^{2}\right)^{2}+\frac{1}{2}\left(\delta m_{2}^{2}+\frac{\delta \lambda_{2}^{A}}{6 N} v^{2}\right) \int_{k}\left(G_{\sigma}(k)+(N-1) G_{\pi}(k)\right)+\frac{\delta \lambda_{2}^{B} v^{2}}{6 N} \int_{k} G_{\sigma}(k) \\
& +\frac{1}{24 N}\left\{\delta \lambda_{0}^{A}\left[\int_{k}\left((N-1) G_{\pi}(k)+G_{\sigma}(k)\right)\right]^{2}+2 \delta \lambda_{0}^{B}\left[(N-1)\left[\int_{k} G_{\pi}(k)\right]^{2}+\left[\int_{k} G_{\sigma}(k)\right]^{2}\right]\right\} \\
& -\frac{\delta Z}{2} \int_{p} p^{2} G_{\sigma}(p)-(N-1) \frac{\delta Z}{2} \int_{p} p^{2} G_{\pi}(p) .
\end{aligned}
$$

The introduction of the two quartic counterterms $\delta \lambda_{0}^{A}$ and $\delta \lambda_{0}^{B}$ corresponds to the two independent $O(N)$-invariant combinations $G_{a b} G_{b a}$ and $G_{a a} G_{b b}$. Similarly $\delta m_{2}^{2}, \delta \lambda_{2}^{A}$, and $\delta \lambda_{2}^{B}$ are introduced to cancel divergences emerging from the two terms, $\delta_{a b} G_{a b}$ and $\hat{v}_{a} \hat{v}_{b} G_{a b}$, appearing in $D_{a b}^{-1} G_{b a}$. 
In the one-component model divergent contributions to the equation of state $\delta V / \delta v=0$ and to the propagator equation $\delta V / \delta G(k)=0$ will be expressed with the divergent pieces of the following integrals:

$$
\begin{aligned}
T[G] & :=\int_{k} G(k)=T_{\operatorname{div}}+T_{F}[G] \\
I(p, G) & :=-i \int_{k} G(k) G(k+p)=I_{\operatorname{div}}+I_{F}(p, G), \\
S(p, G) & :=-i \int_{k} \int_{q} G(k) G(q) G(k+q+p)=S_{\operatorname{div}}(p, G)+S_{F}(p, G) .
\end{aligned}
$$

For the $N$-component case one introduces correspondingly $T_{\alpha}, I_{\alpha \beta}, S_{\alpha \beta \gamma}$, where the indices can take the values $\alpha=\{\pi, \sigma\}$. Separation of the divergent pieces is explicitly presented in the Appendices. Without introducing any particular notation, the finite parts of these integrals would include temperature and density dependent contributions.

The non-perturbative construction of the counterterms will be presented in two stages. First, we restrict the discussion to the two-loop truncation of the effective actions given in (11) and (4). In this case the asymptotic behaviour of the propagator remains as it is at tree-level. In a second step we shall discuss the effect of adding the "basket-ball" diagram, which changes the large momentum asymptotics. We believe that the structured presentation makes more clear the algorithm underlying the somewhat complex formulae and facilitates its prospective numerical implementations.

\section{Analysis of the two-loop truncation: one component real scalar field}

In this truncation one omits the contribution of the last but one term of (1). The equation for the propagator reads as

$$
i G^{-1}(p)=p^{2}-m^{2}-\delta m_{2}^{2}-\frac{1}{2}\left(\lambda+\delta \lambda_{2}\right) v^{2}-\frac{1}{2}\left(\lambda+\delta \lambda_{0}\right) T[G]-\frac{1}{2} \lambda^{2} v^{2} I(p, G) .
$$

The large $p$ asymptotics of the propagator is unchanged compared to the tree-level one, and we parametrise the finite self-energy of the exact propagator as

$$
i G^{-1}(p)=p^{2}-M^{2}-\Pi(p) .
$$

The splitting of the finite self-energy into the two pieces

$$
M^{2}=m^{2}+\frac{\lambda}{2} v^{2}+\frac{\lambda}{2} T_{F}[G], \quad \Pi(p)=\frac{\lambda^{2}}{2} v^{2} I_{F}(p, G), \quad \lim _{p \rightarrow \infty} \frac{\Pi(p)}{p^{2}} \rightarrow 0,
$$

separates the momentum-dependent part of the finite self-energy $(\Pi(p))$, coming from the setting-sun integral of the effective-action (11) and the momentum-independent contribution $\left(M^{2}\right)$ which comes from the other two-loop piece of $V[v, G]$. Although this latter term formally corresponds to the 2PI-Hartree truncation discussed in our previous paper [24], its self-consistent determination takes into account the effect of $\Pi(p)$. 
The divergence cancellation condition which determines the counterterms $\delta m_{2}^{2}, \delta \lambda_{2}$, and $\delta \lambda_{0}$ is obtained by subtracting (10) with the parametrisation (11) from (9) and has the form:

$$
0=\delta m_{2}^{2}+\frac{1}{2} \delta \lambda_{2} v^{2}+\frac{1}{2}\left(\lambda+\delta \lambda_{0}\right) T_{\mathrm{div}}+\frac{1}{2} \delta \lambda_{0} T_{F}[G]+\frac{1}{2} \lambda^{2} v^{2} I_{\mathrm{div}} .
$$

The equation of state reads as

$$
v\left(m^{2}+\delta m_{0}^{2}+\frac{1}{6}\left(\lambda+\delta \lambda_{4}\right) v^{2}+\frac{1}{2}\left(\lambda+\delta \lambda_{2}\right) T[G]+\frac{1}{6} \lambda^{2} S(0, G)\right)=0 .
$$

The cancellation condition for the divergent pieces of this equation amounts to

$$
0=\delta m_{0}^{2}+\frac{1}{6} \delta \lambda_{4} v^{2}+\frac{1}{2}\left(\lambda+\delta \lambda_{2}\right) T_{\operatorname{div}}+\frac{1}{2} \delta \lambda_{2} T_{F}[G]+\frac{1}{6} \lambda^{2} S_{\text {div }}(0, G) .
$$

From this equation one should determine $\delta m_{0}^{2}, \delta \lambda_{4}$ making use of the previously determined expression for $\delta \lambda_{2}$.

The regularised expressions of the counter couplings as determined from (12) and (14) give regularisation independent solutions when used in the regularised equations (9) and (13). In our discussion we shall have in mind cut-off regularisation of the divergent integrals. Since the divergent part of these integrals is separated using propagators which are not sensitive to the infrared features of the theory, their entire zero temperature part will be subtracted.

With help of Appendix A one finds the following expressions for the divergent pieces of the basic integrals:

$$
\begin{gathered}
T_{\mathrm{div}}=T_{d}^{(2)}+\left(M^{2}-M_{0}^{2}\right) T_{d}^{(0)}+\frac{1}{2} \lambda^{2} v^{2} T_{d}^{(I)}, \quad I_{\mathrm{div}}=T_{d}^{(0)}, \\
S_{\mathrm{div}}(0, G)=S_{P V}(0)+3 T_{d}^{(0)} T_{F}+3\left(M^{2}-M_{0}^{2}\right)\left(\left(T_{d}^{(0)}\right)^{2}+T_{d}^{(I)}\right)+\frac{3}{2} \lambda^{2} v^{2}\left(T_{d}^{(I)} T_{d}^{(0)}+T_{d}^{(I, 2)}\right),
\end{gathered}
$$

The cut-off dependent integrals $S_{P V}(0), T_{d}^{(2)}, T_{d}^{(0)}, T_{d}^{(I)}$, and $T_{d}^{(I, 2)}$ are defined in Appendix A. $M_{0}^{2}$ is a free parameter introduced in the auxiliary propagator (91) to ensure the infrared finiteness of all integrals. Exploiting the definition of $M^{2}$ given in (11) when using (15) in (12) and (14), one separates the coefficients of $v^{0}, v^{2}$ and of $T_{F}[G]$ exactly as it was done when we discussed the renormalisation of the 2PI-Hartree approximation [24]. The following six equations arise:

$$
\begin{aligned}
& 0=\delta m_{2}^{2}+\frac{1}{2}\left(\lambda+\delta \lambda_{0}\right)\left[T_{d}^{(2)}+\left(m^{2}-M_{0}^{2}\right) T_{d}^{(0)}\right] \\
& 0=\delta \lambda_{2}+\frac{1}{2} \lambda\left(\lambda+\delta \lambda_{0}\right)\left(T_{d}^{(0)}+\lambda T_{d}^{(I)}\right)+\lambda^{2} T_{d}^{(0)} \\
& 0=\delta \lambda_{0}+\frac{1}{2} \lambda\left(\lambda+\delta \lambda_{0}\right) T_{d}^{(0)}, \\
& 0=\delta m_{0}^{2}+\frac{1}{2}\left(\lambda+\delta \lambda_{2}\right)\left[T_{d}^{(2)}+\left(m^{2}-M_{0}^{2}\right) T_{d}^{(0)}\right]+\frac{1}{2} \lambda^{2}\left(m^{2}-M_{0}^{2}\right)\left(\left(T_{d}^{(0)}\right)^{2}+T_{d}^{(I)}\right)+\frac{1}{6} \lambda^{2} S_{P V}(0), \\
& 0=\delta \lambda_{4}+\frac{3}{2} \lambda\left(\lambda+\delta \lambda_{2}+\lambda^{2} T_{d}^{(0)}\right)\left(T_{d}^{(0)}+\lambda T_{d}^{(I)}\right)+\frac{3}{2} \lambda^{3}\left(T_{d}^{(I)}+\lambda T_{d}^{(I, 2)}\right), \\
& 0=\delta \lambda_{2}+\frac{1}{2} \lambda\left(3 \lambda+\delta \lambda_{2}\right) T_{d}^{(0)}+\frac{1}{2} \lambda^{3}\left(\left(T_{d}^{(0)}\right)^{2}+T_{d}^{(I)}\right)
\end{aligned}
$$




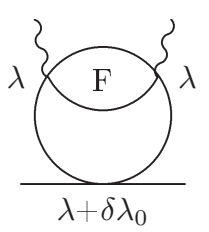

(i)

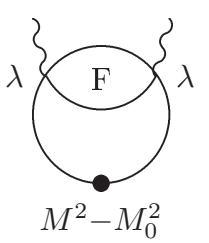

(ii)

Figure 1: Illustration of the appearance of the same radiative structure producing (i) the divergence denoted with $T_{d}^{(I)}$ in the equation for the inverse propagator and (ii) the subdivergence of the equation of state. Internal lines represent the auxiliary propagators $G_{P V}$. Wiggly line represents the background $v$. Letter ' $\mathrm{F}$ ' refers to the finite bubble contribution $I_{P V, F}$.

where the first three equations come from (12), while the last three from (14). The expression for $\delta \lambda_{0}$ determined from the third equation is identical to the expression which we would have obtained in 2PI-Hartree approximation. If one determines from the first five equations $\delta m_{0}^{2}, \delta m_{2}^{2}, \delta \lambda_{0}, \delta \lambda_{2}$, and $\delta \lambda_{4}$, then the sixth serves for a consistency check of the renormalisation. It can be verified by direct substitution of the previously obtained counter coupling expressions. The reason behind this consistency is that when the exact propagator is expanded around the auxiliary propagator the same integral appears both in the momentum independent part of the self-energy and in the contribution of the setting-sun to the equation of state. In the former case the divergent part of this integral denoted with $T_{d}^{(I)}$ appears as an overall divergence proportional to $v^{2}$, which can be seen by looking at the last term of $T_{\mathrm{div}}$ in (15). In the second case it appears also as a subdivergence, that is proportional to $T_{F}[G]$ (see the third term of $S_{\text {div }}$ in (15)). This structural correspondence is illustrated in Fig. 11,

We remark that the results in (16) obtained with the present method reproduce results announced by Arrizabalaga and Reinosa in a conference poster [14] (explicit expressions for the counterterms were given only on the poster, but not in the Proceedings). The use of these expressions in (9) and (13) makes these equations renormalised (cut-off independent).

\section{Adding the "basket-ball" diagram}

Keeping all terms in the $\mathcal{O}\left(\lambda^{2}\right)$ truncation of the skeleton decomposition of the 2PI potential the finite self-energy contains three terms:

$$
\begin{gathered}
i G^{-1}(p)=p^{2}-\Sigma(p), \quad \Sigma(p)=M^{2}+\Pi_{0}(p)+\Pi_{2}(p), \\
M^{2}=m^{2}+\frac{1}{2} \lambda v^{2}+\frac{\lambda}{2} T_{F}[G], \quad \Pi_{0}(p)=\frac{1}{2} \lambda^{2} v^{2} I_{F}(p), \quad \Pi_{2}(p)=\frac{\lambda^{2}}{6} S_{F}(p) .
\end{gathered}
$$

The cancellation of the divergences of the propagator equation amounts to the equality

$$
0=\delta m_{2}^{2}+\frac{1}{2} \delta \lambda_{2} v^{2}+\frac{1}{2}\left(\lambda+\delta \lambda_{0}\right) T_{\mathrm{div}}+\frac{1}{2} \delta \lambda_{0} T_{F}[G]+\frac{1}{2} \lambda^{2} v^{2} I_{\mathrm{div}}+\frac{1}{6} \lambda^{2} S_{\mathrm{div}}(p)-p^{2} \delta Z .
$$

The new feature of the analysis is the fact that the large momentum behaviour of the propagator changes due to the setting-sun contribution. The new leading asymptotics is accompanied also by an infinite wave function renormalisation. The situation is complicated by the fact that the asymptotics 
can be determined only in the course of the numerical solution of the renormalised propagator equation (the equation of state has no influence on the asymptotics). Nevertheless, one proceeds by treating separately the leading asymptotics not depending on any infrared parameter of the theory:

$$
\Pi_{2}(p)=\Pi_{a}(p)+\Pi_{2,0}(p)+\Pi_{r}(p), \quad \lim _{p \rightarrow \infty} \frac{\Pi_{a}(p)}{p^{2}} \sim(\ln p)^{\beta}, \quad \lim _{p \rightarrow \infty} \frac{\Pi_{2,0}(p)}{p^{2}} \rightarrow 0, \quad \Pi_{r}(p) \sim p^{-2} .
$$

The splitting is somewhat arbitrary and this freedom can be exploited to find simple-to-handle expressions for the counter-couplings. There is a corresponding splitting of the setting-sun contribution:

$$
S_{F}(p)=S_{a, F}(p)+S_{0, F}(p)+S_{r}(p),
$$

where $S_{a}(p)$ is defined as the setting-sun diagram computed with a conveniently introduced, infrared stable auxiliary propagator which asymptotically coincides with the exact propagator:

$$
i G_{a}^{-1}(p)=p^{2}-M_{0}^{2}-\Pi_{a}(p), \quad G(p)=G_{a}(p)+\delta G(p), \quad \delta G(p) \sim \mathcal{O}\left(p^{-4}\right) .
$$

Weinberg's theorem [25] guarantees that the self-consistent solution of this equation for the selfenergy (inverse propagator) behaves asymptotically as $\sim p^{2}(\ln p)^{\beta}$, where $\beta$ can be determined only numerically.

Having introduced all the relevant notations we proceed now with the analysis of the divergences. The momentum dependent divergent part of $S_{a}(p)$ is removed by the wave function renormalisation counterterm:

$$
\delta Z=\frac{\lambda^{2}}{6} \frac{\partial}{\partial p^{2}} S_{a}^{\operatorname{div}}(p)
$$

Again, the renormalisation of the bubble integral is the simplest:

$$
I(p)=I_{\mathrm{div}}+I_{F}(p), \quad I_{\mathrm{div}}:=T_{a}^{(0)}, \quad I_{F}(p)=-i \int_{k}\left[G(k) G(k+p)-G_{a}^{2}(k)\right] .
$$

The tadpole integral is analysed with help of the identity given in (102)

$$
T[G]=T_{a}^{(2)}+\left(M^{2}-M_{0}^{2}\right) T_{a}^{(0)}+\frac{1}{2} \lambda^{2} v^{2} T_{a}^{(I)}-i \int_{k} G_{a}^{2}(k) \Pi_{2,0}(k)+T_{F}^{(1)}[G] .
$$

The finite contribution denoted with $T_{F}^{(1)}[G]$ consists of two pieces. The first is the contribution of $G_{r}(p)$ and the second one is the contribution of $I_{F}(k)-I_{a, F}(k)$, which arises when in $\Pi_{0}(k)$ one replaces $I_{F}(k)$ by $I_{a, F}(k)$ to obtain the divergence $T_{a}^{(I)}$. One finds the definitions of $T_{a}^{(2)}, T_{a}^{(0)}$ and of $T_{a}^{(I)}$ in Appendix B.

The determination of $\Pi_{2,0}(k)$ is needed to understand the contribution of the last explicit integral term in the expression of $T[G]$ given in (24). It implies the divergence analysis of $S(p)$. With help of the decomposition $G(p)=G_{a}(p)+\delta G(p)$ (see eq. (102)) one can disentangle the pieces of the setting-sun integral defined in (8), which potentially contain divergences:

$$
S(p)=S_{a}(p)+3 \int_{k} \delta G(k) I_{a}(k+p)+S_{r}^{(1)}(p), \quad S_{r}^{(1)}(p) \sim p^{-2} .
$$

The momentum dependent part of the first term determines the wave function renormalisation, as we have already indicated, and also $\Pi_{a}(p)$. The last term gives a contribution to $\Pi_{r}(p)$. 
One identifies $S_{0, F}(p)$ with the contribution of the integral in (25) which is finite and does not vanish asymptotically for large $p$. Using the expression of $\delta G$ given in (102), simple power counting shows that its $G_{r}(k)$ term gives a second contribution to $\Pi_{r}(p)$. Moreover, in the expression of $\delta G(k)$ one can replace $I_{F}(k)$ by $I_{a, F}(k)$, since the difference gives a third contribution to $\Pi_{r}(p)$. Taking in (25) the finite part of the asymptotic bubble $I_{a}$, one observes that the integral has a logarithmic divergence which is captured by changing $I_{a, F}(k+p)$ to $I_{a, F}(k)$. It is their difference which contributes to $S_{0, F}(p)$. To improve the convergence of the integral in the ultraviolet, one exploits the symmetry property of the integrand and uses instead of $I_{a, F}(k+p)-I_{a, F}(k)$ the kernel

$$
K(p, k)=\frac{\lambda^{2}}{2}\left[I_{a, F}(k+p)+I_{a, F}(k-p)-2 I_{a, F}(k)\right],
$$

which is symmetric under the reflection of $k \rightarrow-k$. Note, that the kernel is not symmetric upon the interchange of $k$ and $p$, that is $K(p, k) \neq K(k, p)$. This is to be contrasted with the symmetric kernel of Ref. [6], denoted with $\Lambda(p, k)$ which, however, is not finite.

With the above steps the following inhomogeneous integral representation is obtained for $S_{0, F}(p)$ :

$$
\frac{\lambda^{2}}{6} S_{0, F}(p)=-\frac{i}{2} \int_{k} G_{a}^{2}(k) K(p, k)\left[M^{2}-M_{0}^{2}+\frac{\lambda^{2}}{2} v^{2} I_{a, F}(k)+\frac{\lambda^{2}}{6} S_{0, F}(k)\right] .
$$

This can be "inverted" iteratively to take the form

$$
\frac{\lambda^{2}}{6} S_{0, F}(p)=\frac{1}{2}\left(M^{2}-M_{0}^{2}\right)\left(-i \int_{k} \Gamma(p, k) G_{a}^{2}(k)\right)+\frac{1}{4} \lambda^{2} v^{2}\left(-i \int_{k} \Gamma(p, k) G_{a}^{2}(k) I_{a, F}(k)\right),
$$

where the kernel $\Gamma(k, p)$ fulfills the Bethe-Salpeter-like equation

$$
\Gamma(p, k)=K(p, k)-\frac{i}{2} \int_{q} G_{a}^{2}(q) K(p, q) \Gamma(q, k) .
$$

The virtue of the representation in (28) is that it decomposes $S_{0, F}(p)$ into the sum of terms proportional to $v^{0}, v^{2}$ and $T_{F}[G]$. It is not a solution for $S_{0, F}$, since $T_{F}[G]$ contains it implicitly, but it means that no new independent finite quantity appears in $\Pi_{2,0}(p)$ relative to the analysis in Section 3. In other words, the subdivergences at this level are found again by looking at the coefficient of $T_{F}[G]$ in the divergence produced by $\Pi_{2,0}(k)=\lambda^{2} S_{0, F}(p) / 6$ through the integral in (24).

Using the expression of $M^{2}$ from (17) in (28) one obtains

$$
\Pi_{2,0}(p)=\frac{1}{2}\left(m^{2}-M_{0}^{2}\right) \Gamma_{0}(p)+\frac{v^{2}}{4}\left[\lambda \Gamma_{0}(p)+\lambda^{2} \Gamma_{1}(p)\right]+\frac{\lambda}{4} T_{F}[G] \Gamma_{0}(p),
$$

where for the finite integrals we introduced the following shorthand notations:

$$
\Gamma_{0}(p)=-i \int_{k} \Gamma(p, k) G_{a}^{2}(k), \quad \Gamma_{1}(p)=-i \int_{k} \Gamma(p, k) G_{a}^{2}(k) I_{a, F}(k) .
$$

The coefficients $\Gamma_{0}(p)$ and $\Gamma_{1}(p)$ multiplying the quantities sensitive to the infrared parameters (e.g. $\left.m^{2}-M_{0}^{2}, v^{2}, T_{F}[G]\right)$ are fully determined by $G_{a}(p)$ and have logarithmic large- $p$ asymptotics!

With the expression of $\Pi_{2,0}(p)$ given in (30) one returns to the expression of the tadpole integral (24). Introducing for the divergences of the remaining integral term the following notations:

$$
D_{0}=-\left.i \int_{k} G_{a}^{2}(k) \Gamma_{0}(k)\right|_{\mathrm{div}}, \quad D_{1}=-\left.i \int_{k} G_{a}^{2}(k) \Gamma_{1}(k)\right|_{\mathrm{div}},
$$


one obtains

$$
\begin{aligned}
T[G]= & T_{a}^{(2)}+\left(m^{2}-M_{0}^{2}\right)\left(T_{a}^{(0)}+\frac{1}{2} D_{0}\right)+\frac{1}{2} \lambda^{2} v^{2}\left[T_{a}^{(0)}+\lambda T_{a}^{(I)}+\frac{1}{2}\left(D_{0}+\lambda D_{1}\right)\right] \\
& +\left[1+\frac{\lambda}{2}\left(T_{a}^{(0)}+\frac{1}{2} D_{0}\right)\right] T_{F}[G] .
\end{aligned}
$$

Note, that the finite parts of the integrals given in (32) are added to $T_{F}^{(1)}[G]$ of (24) to give $T_{F}[G]$. The analysis of the divergences coming from the tadpole integral is now complete.

In order to find the expressions of the counter couplings one needs also $S_{\text {div }}$. Using (102) in (25) one readily obtains:

$$
\begin{aligned}
S_{\text {div }}(p)-\frac{6}{\lambda^{2}} p^{2} \delta Z= & S_{a, \operatorname{div}}(0)+3 T_{a}^{(0)}\left(T[G]-T_{a}^{(2)}\right) \\
& -\left.3 i\left\{\int_{k} G_{a}^{2}(k) I_{a, F}(k+p)\left[M^{2}-M_{0}^{2}+\frac{1}{2} \lambda^{2} v^{2} I_{F}(k)+\Pi_{2,0}(k)\right]\right\}\right|_{\text {div }} \\
= & S_{a, \operatorname{div}}(0)+3 T_{a}^{(0)}\left(T[G]-T_{a}^{(2)}\right)+3\left(M^{2}-M_{0}^{2}\right) T_{a}^{(I)} \\
& +\frac{3}{2} \lambda^{2} v^{2} T_{a}^{(I, 2)}-\left.3 i \int_{k} G_{a}^{2}(k) I_{a, F}(k) \Pi_{2,0}(k)\right|_{\text {div }},
\end{aligned}
$$

where $T[G]$ is given in (33) and the divergences of the last term are expressible using (30). In going from the first to the second form of (34), we are allowed to replace both $I_{F}(k)$ and $I_{a, F}(k+p)$ by $I_{a, F}(k)$ because the differences give finite contributions. It is remarkable that again the infrared quantities $\left(m^{2}, M_{0}^{2}, v^{2}, T_{F}[G]\right)$ appear linearly with coefficients fully determined by $G_{a}$. Therefore the zero temperature part of the integrals determining these coefficients will be fully included into the counterterms!

Having the expressions of $I_{\mathrm{div}}, T_{\mathrm{div}}$, and $S_{\mathrm{div}}$ (see (23), (33), and (34)), one substitutes into the last one the definition of $M^{2}$ from (17) and one proceeds to the decomposition of the divergence cancellation condition for the propagator equation into the three separate equations corresponding to the pieces proportional to $v^{0}, v^{2}$, and $T_{F}[G]$, respectively:

$$
\begin{aligned}
0= & \delta m_{2}^{2}+\frac{1}{2}\left(\lambda+\delta \lambda_{0}\right)\left[T_{a}^{(2)}+\left(m^{2}-M_{0}^{2}\right) T_{a}^{(0)}\right]+\frac{\lambda^{2}}{6} S_{a, \mathrm{div}}(0) \\
& +\left(m^{2}-M_{0}^{2}\right)\left\{\frac{\lambda^{2}}{2}\left[\left(T_{a}^{(0)}\right)^{2}+T_{a}^{(I)}+\frac{1}{2} \tilde{D}_{1}\right]+\frac{1}{4}\left(\lambda+\delta \lambda_{0}+\lambda^{2} T_{a}^{(0)}\right) D_{0}\right\}, \\
0= & \delta \lambda_{2}+\frac{1}{2} \lambda\left(\lambda+\delta \lambda_{0}\right)\left(T_{a}^{(0)}+\lambda T_{a}^{(I)}\right)+\lambda^{2} T_{a}^{(0)} \\
& +\frac{1}{2} \lambda^{3}\left[\left(T_{a}^{(0)}\right)^{2}+T_{a}^{(I)}+\lambda\left(T_{a}^{(0)} T_{a}^{(I)}+T_{a}^{(I, 2)}\right)\right] \\
& +\frac{1}{4} \lambda\left[\left(\lambda+\delta \lambda_{0}+\lambda^{2} T_{a}^{(0)}\right)\left(D_{0}+\lambda D_{1}\right)+\lambda^{2}\left(\tilde{D}_{1}+\lambda D_{2}\right)\right] \\
0= & \delta \lambda_{0}+\frac{1}{2} \lambda\left(\lambda+\delta \lambda_{0}\right) T_{a}^{(0)}+\lambda^{2}\left[T_{a}^{(0)}+\frac{1}{2} \lambda\left(\left(T_{a}^{(0)}\right)^{2}+T_{a}^{(I)}\right)\right] \\
& +\frac{1}{4} \lambda\left[\left(\lambda+\delta \lambda_{0}+\lambda^{2} T_{a}^{(0)}\right) D_{0}+\lambda^{2} \tilde{D}_{1}\right]
\end{aligned}
$$


The new quantities appearing in these equations are the following:

$$
\tilde{D}_{1}=-\left.i \int_{k} G_{a}^{2}(k) I_{a, F}(k) \Gamma_{0}(k)\right|_{\mathrm{div}}, \quad D_{2}=-\left.i \int_{k} G_{a}^{2}(k) I_{a, F}(k) \Gamma_{1}(k)\right|_{\mathrm{div}} .
$$

Note, that $D_{1} \neq \tilde{D}_{1}$ because the kernel $K(p, k)$ given in (26) is asymmetric in its arguments. It should be emphasised that at $\mathcal{O}\left(\lambda^{2}\right)$ one has $\delta \lambda_{0}=\delta \lambda_{2}=-3 \lambda^{2} T_{a}^{(0)} / 2$, as dictated by perturbation theory. Also, one can identify in these equations the contribution from each skeleton diagram separately.

The condition for cancellation of the overall divergences in the equation of state (14) determines $\delta m_{0}^{2}$ and $\delta \lambda_{4}$ :

$$
\begin{aligned}
0= & \delta m_{0}^{2}+\frac{1}{2}\left(\lambda+\delta \lambda_{2}\right)\left[T_{a}^{(2)}+\left(m^{2}-M_{0}^{2}\right) T_{a}^{(0)}\right]+\frac{\lambda^{2}}{6} S_{a, \operatorname{div}}(0) \\
& +\left(m^{2}-M_{0}^{2}\right)\left\{\frac{\lambda^{2}}{2}\left[\left(T_{a}^{(0)}\right)^{2}+T_{a}^{(I)}+\frac{1}{2} \tilde{D}_{1}\right]+\frac{1}{4}\left(\lambda+\delta \lambda_{2}+\lambda^{2} T_{a}^{(0)}\right) D_{0}\right\}, \\
0= & \frac{\delta \lambda_{4}}{3}+\frac{1}{2} \lambda\left(\lambda+\delta \lambda_{2}\right)\left(T_{a}^{(0)}+\lambda T_{a}^{(I)}\right)+\frac{1}{2} \lambda^{3}\left[\left(T_{a}^{(0)}\right)^{2}+T_{a}^{(I)}+\lambda\left(T_{a}^{(0)} T_{a}^{(I)}+T_{a}^{(I, 2)}\right)\right] \\
& +\frac{1}{4} \lambda\left[\left(\lambda+\delta \lambda_{2}+\lambda^{2} T_{a}^{(0)}\right)\left(D_{0}+\lambda D_{1}\right)+\lambda^{2}\left(\tilde{D}_{1}+\lambda D_{2}\right)\right],
\end{aligned}
$$

In addition, the subdivergence cancellation in the equation of state leads to a consistency check between $\delta \lambda_{0}$ and $\delta \lambda_{2}$. The simplest way to obtain it is as follows. Denoting by $T_{\text {div }}\left(T_{F}\right)$ and $S_{0, \text { div }}\left(T_{F}\right)$ the contribution from the tadpole and the setting-sun integrals proportional to $T_{F}[G]$, the subdivergence cancellation in (14) reads as

$$
\frac{1}{2}\left(\lambda+\delta \lambda_{2}\right) T_{\operatorname{div}}\left(T_{F}\right)+\frac{1}{2} \delta \lambda_{2} T_{F}[G]+\frac{1}{6} \lambda^{2} S_{0, \operatorname{div}}\left(T_{F}\right)=0
$$

One should compare this with the condition for subdivergence cancellation in the propagator equation (18):

$$
\frac{1}{2}\left(\lambda+\delta \lambda_{0}\right) T_{\operatorname{div}}\left(T_{F}\right)+\frac{1}{2} \delta \lambda_{0} T_{F}[G]+\frac{1}{6} \lambda^{2} S_{0, \operatorname{div}}\left(T_{F}\right)=0
$$

Using (33), from the comparison it follows that

$$
\left(\delta \lambda_{0}-\delta \lambda_{2}\right)\left[1+\frac{\lambda}{2}\left(T_{a}^{(0)}+\frac{1}{2} D_{0}\right)\right]=0 .
$$

It is not obvious, when one looks at (36) and (37), that this relation is fulfilled, since when one takes from these equations the difference of the two quartic counter couplings, one finds

$$
\begin{aligned}
\delta \lambda_{0}-\delta \lambda_{2} & =\frac{\lambda^{3}}{2}\left[1+\frac{\lambda}{2}\left(T_{a}^{(0)}+\frac{1}{2} D_{0}\right)\right]^{-1}\left\{T_{a}^{(I)}+\frac{1}{2} D_{1}+\lambda\left(T_{a}^{(I, 2)}+\frac{1}{2} D_{2}\right)\right. \\
& \left.+\frac{1}{2} \lambda^{2}\left[\left(T_{a}^{(I, 2)}+\frac{1}{2} D_{2}\right)\left(T_{a}^{(0)}+\frac{1}{2} D_{0}\right)-\left(T_{a}^{(I)}+\frac{1}{2} D_{1}\right)\left(T_{a}^{(I)}+\frac{1}{2} \tilde{D}_{1}\right)\right]\right\} .
\end{aligned}
$$

Comparing (43) and (44) one has

$$
\delta \lambda_{0}=\delta \lambda_{2}
$$


and the requirement of the vanishing of the expression in the curly bracket of (44). This condition relates several cut-off dependent integrals which can be (and should be) checked only when the numerical solutions for $G(p)$ and for the Bethe-Salpeter-like equation (29) are found.

With a fully analogous line of thinking one can investigate the consistency of the cancellation conditions of the divergences proportional with $v^{0}$ and $v^{2}$ in the propagator equation and the equation of state. Using (43) the consistency requirement leads to the relations:

$$
\delta m_{2}^{2}=\delta m_{0}^{2}, \quad \delta \lambda_{4}=3\left(\delta \lambda_{2}+\lambda^{2} T_{a}^{(0)}\right) .
$$

These two relations can be obtained also by comparing (35) with (39) and (36) with (40). Since $\delta \lambda_{0}=-3 \lambda^{2} T_{a}^{(0)} / 2$, to $\mathcal{O}\left(\lambda^{2}\right)$ all three quartic coupling counterterms are equal, as is expected from perturbative renormalisation.

\section{Analysis of the $O(N)$ model at the two-loop level of the truncation}

The broken symmetry phase of the $O(N)$ model represents a non-trivial test-ground for checking the consistency of our counterterm determination. It has two independent excitations: the $(N-1)$-fold degenerate "pion" multiplet and the more massive "sigma". Therefore there are four renormalisability conditions for each of the two propagators. Since from the counterterms introduced in Section 2 only five enter the two propagator equations, one has to check if the remaining three conditions are fulfilled. Although the algebra of this calculation is quite challenging with help of symbolic computer calculations we could check that the extra conditions do not impose any new relation on the counterterms, being identically satisfied.

The equations for the inverse propagators $i G_{\sigma}^{-1}(p)=p^{2}-\Sigma_{0}^{\sigma}(p)$ and $i G_{\pi}^{-1}(p)=p^{2}-\Sigma_{0}^{\pi}(p)$ lead to the following equations for the respective self-energies:

$$
\begin{aligned}
\Sigma_{0}^{\pi}(p)= & m^{2}+\delta m_{2}^{2}+\left(\lambda+\delta \lambda_{2}^{A}\right) \frac{v^{2}}{6 N}+\frac{1}{6 N}\left[(N+1) \lambda+(N-1) \delta \lambda_{0}^{A}+2 \delta \lambda_{0}^{B}\right] T_{\pi}+\frac{1}{6 N}\left(\lambda+\delta \lambda_{0}^{A}\right) T_{\sigma} \\
& +\frac{\lambda^{2} v^{2}}{9 N^{2}} I_{\sigma \pi}(p), \\
\Sigma_{0}^{\sigma}(p)= & m^{2}+\delta m_{2}^{2}+\left(3 \lambda+\delta \lambda_{2}^{A}+2 \delta \lambda_{2}^{B}\right) \frac{v^{2}}{6 N}+\frac{1}{6 N}\left(3 \lambda+\delta \lambda_{0}^{A}+2 \delta \lambda_{0}^{B}\right) T_{\sigma}+\frac{N-1}{6 N}\left(\lambda+\delta \lambda_{0}^{A}\right) T_{\pi} \\
& +\frac{\lambda^{2} v^{2}}{18 N^{2}}\left[9 I_{\sigma \sigma}(p)+(N-1) I_{\pi \pi}(p)\right] .
\end{aligned}
$$

Here one ought to recall that the pion propagator determined self-consistently in the 2PI approximation does not obey Goldstone's theorem e.g. $\Sigma_{0}^{\pi}(p=0) \neq 0$. There exists a proposition at Hartree level [26] to modify the 2PI functional to cure this deficiency, which one might attempt to generalise when including $\mathcal{O}\left(\lambda^{2}\right)$ skeleton diagrams. The method of counterterm resummation presented here would work equally well for such modified 2PI functionals.

When looking at (48), one can make the following identifications of the respective finite tadpole and bubble contributions:

$$
M_{\pi}^{2}=m^{2}+\frac{\lambda}{6 N} v^{2}+\frac{\lambda}{6 N}\left[T_{\sigma, F}+(N+1) T_{\pi, F}\right], \quad \Pi_{0}^{\pi}(p)=\frac{\lambda^{2} v^{2}}{9 N^{2}} I_{\sigma \pi}^{F}(p),
$$




$$
M_{\sigma}^{2}=m^{2}+\frac{\lambda}{2 N} v^{2}+\frac{\lambda}{6 N}\left[3 T_{\sigma, F}+(N-1) T_{\pi, F}\right], \quad \Pi_{0}^{\sigma}(p)=\frac{\lambda^{2} v^{2}}{18 N^{2}}\left[9 I_{\sigma \sigma}^{F}(p)+(N-1) I_{\pi \pi}^{F}(p)\right] .
$$

The conditions for the renormalisability of the two self-energies $\Sigma_{0}^{\pi}(p)$ and $\Sigma_{0}^{\sigma}(p)$ read as

$$
\begin{aligned}
0= & \delta m_{2}^{2}+\frac{\delta \lambda_{2}^{A}}{6 N} v^{2}+\frac{1}{6 N}\left[(N+1) \lambda+(N-1) \delta \lambda_{0}^{A}+2 \delta \lambda_{0}^{B}\right] T_{\pi, \mathrm{div}}+\frac{1}{6 N}\left(\lambda+\delta \lambda_{0}^{A}\right) T_{\sigma, \mathrm{div}} \\
& +\frac{1}{6 N}\left[(N-1) \delta \lambda_{0}^{A}+2 \delta \lambda_{0}^{B}\right] T_{\pi, F}+\frac{\delta \lambda_{0}^{A}}{6 N} T_{\sigma, F}+\frac{\lambda^{2} v^{2}}{9 N^{2}} T_{d}^{(0)}, \\
0= & \delta m_{2}^{2}+\left(\delta \lambda_{2}^{A}+2 \delta \lambda_{2}^{B}\right) \frac{v^{2}}{6 N}+\frac{1}{6 N}\left(3 \lambda+\delta \lambda_{0}^{A}+2 \delta \lambda_{0}^{B}\right) T_{\sigma, \mathrm{div}}+\frac{N-1}{6 N}\left(\lambda+\delta \lambda_{0}^{A}\right) T_{\pi, \mathrm{div}} \\
& +\frac{1}{6 N}\left(\delta \lambda_{0}^{A}+2 \delta \lambda_{0}^{B}\right) T_{\sigma, F}+\frac{N-1}{6 N} \delta \lambda_{0}^{A} T_{\pi, F}+\frac{\lambda^{2} v^{2}}{18 N^{2}}(N+8) T_{d}^{(0)} .
\end{aligned}
$$

For the three bubble integrals we used the analogue of (92) and the fact that their divergence does not depend on the kind of fields which constitute them.

In analogy with the case of the one-component scalar field, one can write down the explicit expressions for $T_{\pi, \text { div }}$ and $T_{\sigma, \text { div }}$ expanding, as in (93), the exact propagators in the tadpole integrals around the common auxiliary propagator. One obtains

$$
\begin{aligned}
& T_{\pi, \mathrm{div}}=T_{d}^{(2)}+\left(M_{\pi}^{2}-M_{0}^{2}\right) T_{d}^{(0)}+\frac{\lambda^{2} v^{2}}{9 N^{2}} T_{\pi, d}^{\sigma \pi(I)}, \\
& T_{\sigma, \mathrm{div}}=T_{d}^{(2)}+\left(M_{\sigma}^{2}-M_{0}^{2}\right) T_{d}^{(0)}+\frac{\lambda^{2} v^{2}}{2 N^{2}} T_{\sigma, d}^{\sigma \sigma(I)}+\frac{\lambda^{2} v^{2}}{18 N^{2}}(N-1) T_{\sigma, d}^{\pi \pi(I)} .
\end{aligned}
$$

Although, the divergence involving integrands proportional to the finite bubbles $I_{\alpha \beta}^{F}$ does not depend on the kind of fields which constitute them, in the notation above we explicitly keep track of the bubble which produces the divergence. The upper double index gives the two propagators constituting the bubble, while lower index refers to the self-energy they contribute to. This distinction will be exploited at the end of this section to generalise, to the present case, the diagrammatic interpretation of the consistency of the renormalisation conditions given at the end of Section 3.

Substituting (52) and (53) into the renormalisation conditions, one uses the defining equations of $M_{\sigma}^{2}$ and $M_{\pi}^{2}$ and requires again separate vanishing of the coefficients of the divergent pieces proportional to $v^{0}, v^{2}, T_{\pi, F}$, and $T_{\sigma, F}$, respectively. For the pion propagator one finds:

$$
\begin{aligned}
0= & \delta m_{2}^{2}+\frac{1}{6 N}\left[(N+2) \lambda+N \delta \lambda_{0}^{A}+2 \delta \lambda_{0}^{B}\right]\left[T_{d}^{(2)}+\left(m^{2}-M_{0}^{2}\right) T_{d}^{(0)}\right] \\
0= & \delta \lambda_{2}^{A}+\left[(N+1) \lambda+(N-1) \delta \lambda_{0}^{A}+2 \delta \lambda_{0}^{B}\right]\left[\frac{\lambda}{6 N} T_{d}^{(0)}+\frac{\lambda^{2}}{9 N^{2}} T_{\pi, d}^{\sigma, \pi(I)}\right] \\
& +\left(\lambda+\delta \lambda_{0}^{A}\right)\left[\frac{\lambda}{2 N} T_{d}^{(0)}+\frac{\lambda^{2}}{2 N^{2}} T_{\sigma, d}^{\sigma \sigma(I)}+\frac{\lambda^{2}}{18 N^{2}}(N-1) T_{\sigma, d}^{\pi \pi(I)}\right]+\frac{2 \lambda^{2}}{3 N} T_{d}^{(0)}, \\
0= & \left.(N-1) \delta \lambda_{0}^{A}+2 \delta \lambda_{0}^{B}+\frac{\lambda}{6 N}\left\{(N-1)\left[(N+2)\left(\lambda+\delta \lambda_{0}^{A}\right)+2\left(\lambda+\delta \lambda_{0}^{B}\right)\right]+4\left(\lambda+\delta \lambda_{0}^{B}\right)\right\} T_{d}^{(0)},\left(56 \lambda_{0}^{A}\right)+2\left(\lambda+\delta \lambda_{0}^{B}\right)\right] T_{d}^{(0)} . \\
0= & \delta \lambda_{0}^{A}+\frac{\lambda}{6 N}\left[( N + 2 ) \left(\lambda+\delta \lambda_{0}\right.\right.
\end{aligned}
$$

The last two equations form a closed set for $\delta \lambda_{0}^{A}$ and $\delta \lambda_{0}^{B}$. Their expressions can be used in the first two equations to obtain $\delta m_{2}^{2}$ and $\delta \lambda_{2}^{A}$. 
In case of the sigma propagator, the corresponding set of renormalisation conditions reproduces exactly the same equation for the mass-renormalisation $\delta m_{2}^{2}$. The condition for vanishing of the divergent coefficients of $T_{\pi, F}$ and $T_{\sigma, F}$ produces formally different expressions when compared to those in (56) and (57), but their solution for $\delta \lambda_{0}^{A}$ and $\delta \lambda_{0}^{B}$ coincides with the solution of (56) and (57). The only new condition arises from the vanishing of the coefficient of $v^{2}$, which reads as

$$
\begin{gathered}
0=\delta \lambda_{2}^{A}+2 \delta \lambda_{2}^{B}+\frac{\lambda}{6 N} T_{d}^{(0)}\left[2(N+8) \lambda+(N+2)\left(\lambda+\delta \lambda_{0}^{A}\right)+6\left(\lambda+\delta \lambda_{0}^{B}\right)\right] \\
+\frac{\lambda^{2}}{18 N^{2}}\left\{2\left(\lambda+\delta \lambda_{0}^{B}\right)\left[(N-1) T_{\sigma, d}^{\pi \pi(I)}+9 T_{\sigma, d}^{\sigma \sigma(I)}\right]\right. \\
\left.+\left(\lambda+\delta \lambda_{0}^{A}\right)\left[(N-1)\left(T_{\sigma, d}^{\pi \pi(I)}+2 T_{\sigma, d}^{\sigma \pi(I)}\right)+9 T_{\sigma, d}^{\sigma \sigma(I)}\right]\right\} .
\end{gathered}
$$

Using the expression of $\delta \lambda_{2}^{A}$ obtained from (55) the equation above determines $\delta \lambda_{2}^{B}$. Note, that the expressions for $\delta \lambda_{0}^{A}, \delta \lambda_{0}^{B}$ and $\delta m_{2}^{2}$ are identical with those obtained in the 2PI-Hartree approximation in [24]. The extra term in the effective action, compared to the 2PI-Hartree approximation is responsible for the fact that $\delta \lambda_{2}^{A} \neq \delta \lambda_{0}^{A}$ and $\delta \lambda_{2}^{B} \neq \delta \lambda_{0}^{B}$.

In the broken symmetry phase the equation of state is given by

$$
\begin{aligned}
0= & m^{2}+\delta m_{0}^{2}+\frac{1}{6 N}\left(\lambda+\delta \lambda_{4}\right) v^{2}+\frac{N-1}{6 N}\left(\lambda+\delta \lambda_{2}^{A}\right) T_{\pi}+\frac{1}{6 N}\left(3 \lambda+\delta \lambda_{2}^{A}+2 \lambda_{2}^{B}\right) T_{\sigma} \\
& +\frac{\lambda^{2}}{18 N^{2}}\left[3 S_{\sigma \sigma \sigma}(0)+(N-1) S_{\sigma \pi \pi}(0)\right] .
\end{aligned}
$$

The renormalisation conditions emerging from this equation naturally separate into two groups. The vanishing of the divergent coefficients proportional to $v^{0}$ and $v^{2}$ provides the expressions of $\delta m_{0}^{2}$ and $\delta \lambda_{4}$. The consistent renormalisability of the present truncation of the 2PI-approximation depends on the vanishing of the subdivergences proportional to $T_{\pi, F}$ and $T_{\sigma, F}$. There are no extra free parameters to ensure this.

In order to explicitly write the renormalisation conditions, one needs the divergences of the two setting-sun integrals. To obtain them, one does the same steps as in (93) and derives expressions similar to (94) for $\delta G_{\sigma}(p)=G_{\sigma}(p)-G_{P V}(p)$ and $\delta G_{\pi}(p)=G_{\pi}(p)-G_{P V}(p)$, namely:

$$
\begin{aligned}
& \delta G_{\sigma}(p)=i G_{P V}^{2}(p)\left[M_{0}^{2}-M_{\sigma}^{2}-\frac{\lambda^{2} v^{2}}{18 N^{2}}\left(9 I_{\sigma \sigma}^{F}(p)+(N-1) I_{\pi \pi}^{F}(p)\right)\right]+G_{\sigma}^{r}(p), \\
& \delta G_{\pi}(p)=i G_{P V}^{2}(p)\left(M_{0}^{2}-M_{\pi}^{2}-\frac{\lambda^{2} v^{2}}{9 N^{2}} I_{\sigma \pi}^{F}(p)\right)+G_{\pi}^{r}(p) .
\end{aligned}
$$

The regular parts $G_{\sigma}^{r}(p)$ and $G_{\pi}^{r}(p)$ behave asymptotically as $p^{-6}$. Using $G_{\alpha}(p)=G_{P V}(p)+\delta G_{\alpha}(p)$ with $\alpha=\{\sigma, \pi\}$, one finds the following expressions for the two types of setting-sun integrals in which the parts contributing to the divergences are well separated:

$$
\begin{aligned}
& S_{\sigma \sigma \sigma}(0)=S_{P V}(0)+3 \int_{k} \delta G_{\sigma}(k) I_{P V}^{\sigma \sigma}(k)+S_{\sigma \sigma \sigma}^{F(1)}, \\
& S_{\sigma \pi \pi}(0)=S_{P V}(0)+\int_{k} \delta G_{\sigma}(k) I_{P V}^{\pi \pi}(k)+2 \int_{k} \delta G_{\pi}(k) I_{P V}^{\sigma \pi}(k)+S_{\sigma \pi \pi}^{F(1)} .
\end{aligned}
$$

For the bubble $I_{P V}(k)$ we indicated the original two propagators which were replaced by auxiliary ones. 
When substituting (60) and (61) into (62) one replaces $I_{\alpha \beta}^{F}(p)$ with $I_{P V, F}^{\alpha \beta}(p)(\alpha \in\{\pi, \sigma\})$, since the difference gives a finite contribution. The same steps as performed on (97) give for the divergent parts of the setting-sun diagrams:

$$
\begin{aligned}
S_{\sigma \sigma \sigma}^{\mathrm{div}}(0)= & S_{P V}(0)+3 T_{d}^{(0)}\left(T_{\sigma, F}-T_{d}^{(2)}\right)+3\left(M_{\sigma}^{2}-M_{0}^{2}\right) T_{\sigma, d}^{\sigma \sigma(I)}+\frac{\lambda^{2} v^{2}}{6 N^{2}}(N+8) T_{d}^{(I, 2)}, \\
S_{\sigma \pi \pi}^{\operatorname{div}}(0)= & S_{P V}(0)+T_{d}^{(0)}\left(T_{\sigma, F}+2 T_{\pi, F}-3 T_{d}^{(2)}\right)+\left(M_{\sigma}^{2}-M_{0}^{2}\right) T_{\sigma, d}^{\pi(I)}+2\left(M_{\pi}^{2}-M_{0}^{2}\right) T_{\pi, d}^{\sigma \pi(I)} \\
& +\frac{\lambda^{2} v^{2}}{18 N^{2}}(N+12) T_{d}^{(I, 2)} .
\end{aligned}
$$

The divergent expressions $T_{\sigma, d}^{\sigma \sigma(I)}, T_{\sigma, d}^{\pi \pi(I)}$, and $T_{\pi, d}^{\sigma \pi(I)}$ are the same as those occurring in (52) and (53). For the divergent part of the integral containing two bubbles we used the definition (99) without distinguishing which bubble produces it, because this is an overall divergence and does not show up in any consistency condition.

The condition for cancellation of the divergences in the equation of state (59) reads as

$$
\begin{aligned}
0= & \delta m_{0}^{2}+\frac{\delta \lambda_{4}}{6 N} v^{2}+\frac{N-1}{6 N}\left(\lambda+\delta \lambda_{2}^{A}\right) T_{\pi, \operatorname{div}}+\frac{N-1}{6 N} \delta \lambda_{2}^{A} T_{\pi, F} \\
& +\left(3 \lambda+\delta \lambda_{2}^{A}+2 \delta \lambda_{2}^{B}\right) \frac{T_{\sigma, \mathrm{div}}}{6 N}+\left(\delta \lambda_{2}^{A}+2 \delta \lambda_{2}^{B}\right) \frac{T_{\sigma, F}}{6 N}+\frac{\lambda^{2}}{18 N^{2}}\left[3 S_{\sigma \sigma \sigma}^{\mathrm{div}}(0)+(N-1) S_{\sigma \pi \pi}^{\mathrm{div}}(0)\right]
\end{aligned}
$$

The renormalisation conditions can be read from this equation using the expressions given in (52), (53), (63), and (64) with the corresponding formulae for $M_{\sigma}^{2}$ and $M_{\pi}^{2}$ given in (49). The counterterms $\delta m_{0}^{2}$ and $\delta \lambda_{4}$ are determined from the vanishing of the coefficients of $v^{0}$ and $v^{2}$, respectively. The conditions for the cancellation of the coefficients of $T_{\sigma, F}$ and $T_{\pi, F}$ are given by

$$
\begin{aligned}
0= & \frac{1}{6 N}\left(\delta \lambda_{2}^{A}+2 \delta \lambda_{2}^{B}\right)+\frac{\lambda T_{d}^{(0)}}{36 N^{2}}\left[3 \lambda(N+8)+(N+2) \delta \lambda_{2}^{A}+6 \delta \lambda_{2}^{B}\right]+\frac{\lambda^{3}\left(T_{d}^{(0)}\right)^{2}}{108 N^{3}}(5 N+22) \\
& +\frac{\lambda^{3}}{N^{3}}\left(\frac{1}{4} T_{\sigma, d}^{\sigma \sigma(I)}+\frac{N-1}{36} T_{\sigma, d}^{\pi \pi(I)}+\frac{N-1}{54} T_{\pi, d}^{\sigma \pi(I)}\right), \\
0= & \frac{\delta \lambda_{2}^{A}}{6 N}+\frac{\lambda T_{d}^{(0)}}{36 N^{2}}\left[\lambda(N+8)+(N+2) \delta \lambda_{2}^{A}+2 \delta \lambda_{2}^{B}\right]+\frac{\lambda^{3}\left(T_{d}^{(0)}\right)^{2}}{108 N^{3}}(3 N+10) \\
& +\frac{\lambda^{3}}{6 N^{3}}\left(\frac{1}{2} T_{\sigma, d}^{\sigma \sigma(I)}+\frac{N-1}{18} T_{\sigma, d}^{\pi \pi(I)}+\frac{N+1}{9} T_{\pi, d}^{\sigma \pi(I)}\right) .
\end{aligned}
$$

Using the solution for $\delta \lambda_{2}^{A}$ and $\delta \lambda_{2}^{B}$, as obtained from (55) and (158), and the solution for $\delta \lambda_{0}^{A}$ and $\delta \lambda_{0}^{B}$ obtained from (56) and (57), one can easily verify that the above two consistency conditions are fulfilled. Note, that the coefficients of $T_{\sigma, d}^{\sigma \sigma(I)}, T_{\sigma, d}^{\pi \pi(I)}$, and $T_{\pi, d}^{\sigma \pi(I)}$ separately vanish when the solution for the coupling counterterms are used. The reason is the same as the one given at the end of Section 3, namely the appearance of the same structure in the renormalisation conditions of both the propagator equations and of the equation of state. The diagrammatic illustration given in Fig. 2 parallels that of Fig. 1. Diagrams (a), (b) and diagram (c) correspond to the last two terms of (53) and the last term of (52), respectively, while diagram (d) and diagrams (e) and (f) correspond to the last but one term in (63) and the last two terms of the first line of (64), respectively. 


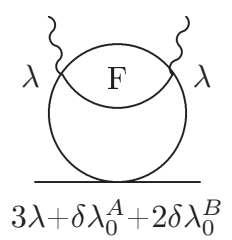

(a)

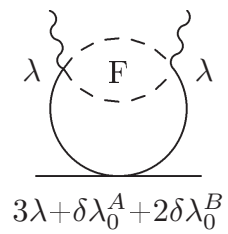

(b)

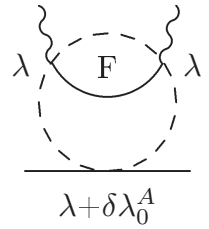

(c)

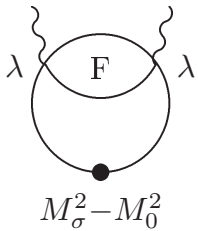

(d)

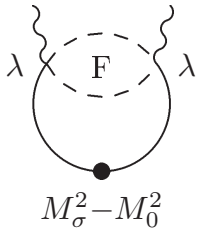

(e)

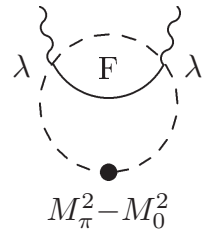

(f)

Figure 2: Illustration of the appearance of the same radiative structures producing the divergences denoted by $T_{\sigma, d}^{\sigma \sigma(I)}, T_{\sigma, d}^{\pi \pi(I)}$, and $T_{\pi, d}^{\sigma \pi(I)}$ in the equation for the sigma propagator and the subdivergences of the equation of state. Solid (dashed) lines indicate that the auxiliary propagator $G_{P V}$ comes from a (pseudo)scalar propagator. For other notations we refer to Fig. 1.

\section{Analysis of the $O(N)$ model with "basket-ball" diagrams included}

At the $\mathcal{O}\left(\lambda^{2}\right)$ truncation level of the effective action the finite self-energies are decomposed as

$$
\begin{array}{ll}
\Sigma_{\pi}(p)=M_{\pi}^{2}+\Pi_{0}^{\pi}(p)+\Pi_{2}^{\pi}(p), & \Pi_{2}^{\pi}(p)=\frac{\lambda^{2}}{18 N^{2}}\left[S_{\sigma \sigma \pi}^{F}(p)+(N+1) S_{\pi \pi \pi}^{F}(p)\right], \\
\Sigma_{\sigma}(p)=M_{\sigma}^{2}+\Pi_{0}^{\sigma}(p)+\Pi_{2}^{\sigma}(p), & \Pi_{2}^{\sigma}(p)=\frac{\lambda^{2}}{18 N^{2}}\left[3 S_{\sigma \sigma \sigma}^{F}(p)+(N-1) S_{\sigma \pi \pi}^{F}(p)\right],
\end{array}
$$

where $M_{\pi}^{2}, M_{\sigma}^{2}, \Pi_{0}^{\pi}(p)$ and $\Pi_{0}^{\sigma}(p)$ are defined in (49). Since the infrared sector has no influence on the asymptotic behaviour of the self-energies, one can separate a common leading asymptotics in the part of the self-energies containing the contribution of the setting-sun integrals $(\alpha \in\{\pi, \sigma\})$ :

$$
\Pi_{2}^{\alpha}(p)=\Pi_{a}(p)+\Pi_{2,0}^{\alpha}(p)+\Pi_{r}^{\alpha}(p), \quad \lim _{p \rightarrow \infty} \frac{\Pi_{a}(p)}{p^{2}} \sim(\ln p)^{\beta}, \quad \lim _{p \rightarrow \infty} \frac{\Pi_{2,0}^{\alpha}(p)}{p^{2}} \rightarrow 0, \quad \Pi_{r}^{\alpha}(p) \sim p^{-2} .
$$

The corresponding decomposition of the finite contribution of the setting-sun integrals reads as

$$
S_{\alpha \beta \beta}^{F}(p)=S_{a, F}(p)+S_{\alpha \beta \beta}^{0, F}(p)+S_{\alpha \beta \beta}^{r}(p),
$$

where $\alpha, \beta \in\{\pi, \sigma\}$.

Compared to (501) and (51) the renormalisation conditions for the equations of $G_{\pi}^{-1}(p)$ and $G_{\sigma}^{-1}(p)$ are now completed, respectively, by the following terms:

$$
-p^{2} \delta Z+\Sigma_{\pi}^{\operatorname{div}}(p), \quad-p^{2} \delta Z+\Sigma_{\sigma}^{\operatorname{div}}(p),
$$

where

$$
\Sigma_{\pi}^{\mathrm{div}}(p)=\frac{\lambda^{2}}{18 N^{2}}\left[S_{\sigma \sigma \pi}^{\mathrm{div}}(p)+(N+1) S_{\pi \pi \pi}^{\mathrm{div}}(p)\right], \quad \Sigma_{\sigma}^{\mathrm{div}}(p)=\frac{\lambda^{2}}{18 N^{2}}\left[3 S_{\sigma \sigma \sigma}^{\mathrm{div}}(p)+(N-1) S_{\sigma \pi \pi}^{\mathrm{div}}(p)\right] .
$$

The renormalisation condition (65) for the equation of state remains unchanged. Evidently, in (50) and (51) one has to replace $T_{d}^{(0)}$ by $T_{a}^{(0)}$. 
With the same steps which lead from (101) to (102) one can write the following representation for the propagators

$$
G_{\alpha}(p)=G_{a}(p)+\delta G_{\alpha}(p), \quad \delta G_{\alpha}(p):=-i G_{a}^{2}(p)\left[M_{\alpha}^{2}-M_{0}^{2}+\Pi_{0}^{\alpha}(p)+\Pi_{2,0}^{\alpha}(p)\right]+G_{r}^{\alpha}(p),
$$

where $G_{r}^{\alpha}(p)$ behaves asymptotically as $p^{-6}$.

Using (74), the divergences of the tadpole integrals can be easily separated

$$
\begin{aligned}
& T_{\sigma}=T_{a}^{(2)}+\left(M_{\sigma}^{2}-M_{0}^{2}\right) T_{a}^{(0)}+\frac{\lambda^{2} v^{2}}{18 N^{2}}(N+8) T_{a}^{(I)}-\left.i \int_{k} G_{a}^{2}(k) \Pi_{2,0}^{\sigma}(k)\right|_{\mathrm{div}}+T_{\sigma, F}, \\
& T_{\pi}=T_{a}^{(2)}+\left(M_{\pi}^{2}-M_{0}^{2}\right) T_{a}^{(0)}+\frac{\lambda^{2} v^{2}}{9 N^{2}} T_{a}^{(I)}-\left.i \int_{k} G_{a}^{2}(k) \Pi_{2,0}^{\pi}(k)\right|_{\mathrm{div}}+T_{\pi, F} .
\end{aligned}
$$

The same procedure which led to (27) provides now an integral equation for the finite piece of the setting-sun integrals $S_{\alpha \beta \beta}^{0, F}(p)$ which grows no faster than logarithmically for asymptotic values of $p$. Combining the corresponding contributions of different setting-sun integrals one obtains the following set of coupled integral representations:

$$
\begin{aligned}
& \Pi_{2,0}^{\sigma}(p)=-\frac{i}{18 N^{2}} \int_{k} G_{a}^{2}(k) K(p, k)\left[F_{\sigma}(k)+A_{\sigma \sigma} \Pi_{2,0}^{\sigma}(k)+A_{\sigma \pi} \Pi_{2,0}^{\pi}(k)\right], \\
& \Pi_{2,0}^{\pi}(p)=-\frac{i}{18 N^{2}} \int_{k} G_{a}^{2}(k) K(p, k)\left[F_{\pi}(k)+A_{\pi \sigma} \Pi_{2,0}^{\sigma}(k)+A_{\pi \pi} \Pi_{2,0}^{\pi}(k)\right] .
\end{aligned}
$$

$K(p, k)$ was defined in (26). Here we introduced the matrix $A$ with elements $A_{\sigma \sigma}=N+8, A_{\sigma \pi}=$ $2(N-1), A_{\pi \sigma}=2, A_{\pi \pi}=3 N+4$, and also the two inhomogeneous terms of the above relations:

$$
\begin{aligned}
& F_{\sigma}(k)=A_{\sigma \sigma}\left(M_{\sigma}^{2}-M_{0}^{2}\right)+A_{\sigma \pi}\left(M_{\pi}^{2}-M_{0}^{2}\right)+\frac{\lambda^{2} v^{2}}{18 N^{2}} B_{\sigma} I_{a, F}(k), \\
& F_{\pi}(k)=A_{\pi \sigma}\left(M_{\pi}^{2}-M_{0}^{2}\right)+A_{\pi \pi}\left(M_{\pi}^{2}-M_{0}^{2}\right)+\frac{\lambda^{2} v^{2}}{18 N^{2}} B_{\pi} I_{a, F}(k),
\end{aligned}
$$

with $B_{\sigma}=(N+8)^{2}+4(N-1)$ and $B_{\pi}=8(N+3)$.

The solution of (76) takes the form

$$
\Pi_{2,0}^{\alpha}(p)=-\frac{i}{18 N^{2}} \int_{k} G_{a}^{2}(k) \Gamma_{\alpha \beta}(p, k)\left[A_{\beta \gamma}\left(M_{\gamma}^{2}-M_{0}^{2} u_{\gamma}\right)+\frac{\lambda^{2}}{18 N^{2}} B_{\beta} I_{a, F}(k)\right],
$$

where $\alpha, \beta, \gamma \in\{\pi, \sigma\}, u_{\pi}=u_{\sigma}=1$. $\Gamma_{\alpha \beta}(p, k)$ satisfies the following Bethe-Salpeter-like matrix equation

$$
\Gamma_{\alpha \beta}(p, k)=K(p, k) \delta_{\alpha \beta}-\frac{i}{18 N^{2}} \int_{q} K(p, q) A_{\alpha \gamma} \Gamma_{\gamma \beta}(q, k) .
$$

One observes that $\Gamma_{\sigma \sigma}(p, k)$ couples only to $\Gamma_{\pi \sigma}(p, k)$ and $\Gamma_{\pi \pi}(p, k)$ couples to $\Gamma_{\sigma \pi}(p, k)$. Exploiting that $A_{\sigma \sigma}-A_{\pi \sigma}=A_{\pi \pi}-A_{\sigma \pi}$, one can verify that the two combinations $Q_{1}(p, k)=\Gamma_{\sigma \sigma}(p, k)-\Gamma_{\pi \sigma}(p, k)$ and $Q_{2}(p, k)=\Gamma_{\pi \pi}(p, k)-\Gamma_{\sigma \pi}(p, k)$ satisfy the same equation

$$
Q_{i}(p, k)=K(p, k)-c \frac{i}{18 N^{2}} \int_{q} K(p, q) Q_{i}(q, k)
$$


where $c=N+6$. This means that $Q_{1}(p, k)=Q_{2}(p, k)$, and consequently there is a relation between the elements of the $\Gamma(p, k)$ matrix:

$$
\Gamma_{\sigma \sigma}(p, k)-\Gamma_{\pi \sigma}(p, k)=\Gamma_{\pi \pi}(p, k)-\Gamma_{\sigma \pi}(p, k) .
$$

Another relation which can be derived reads as

$$
\Gamma_{\sigma \sigma}(p, k)+(N-1) \Gamma_{\pi \sigma}(p, k)=\Gamma_{\pi \pi}(p, k)+(N-1)^{-1} \Gamma_{\sigma \pi}(p, k) .
$$

This is because the combinations on the left- and right-hand sides of the equation both satisfy an equation similar to (80), but with $c$ replaced by $\tilde{c}=3(N+2)$. Making use of (81) in (82) one obtains

$$
\Gamma_{\sigma \pi}(p, k)=(N-1) \Gamma_{\pi \sigma}(p, k) .
$$

We shall see, that the relations (81) and (83) play an important role in checking some of the consistency relations of our renormalisation procedure.

Having obtained the integral representation (78) for $\Pi_{2,0}^{\sigma}(p)$ and $\Pi_{2,0}^{\pi}(p)$, one uses them in (75) to write the divergent part of the tadpole integrals with help of the new divergent matrices $D_{i}^{\alpha \gamma}$ to be defined below:

$$
T_{\alpha, \text { div }}=T_{a}^{(2)} u_{\alpha}+\left(T_{a}^{(0)} \delta_{\alpha \gamma}+D_{0}^{\alpha \gamma} A_{\beta \gamma}\right)\left(M_{\gamma}^{2}-M_{0}^{2} u_{\gamma}\right)+\frac{\lambda^{2} v^{2}}{18 N^{2}}\left(T_{a}^{(I)} A_{\alpha \sigma}+D_{1}^{\alpha \beta} B_{\beta}\right) .
$$

The same new divergent quantities appear in the divergent part of the self-energies containing the contributions of the setting-sun integrals. One obtains with the procedure which gave (34) the following expression:

$$
\begin{aligned}
\Sigma_{\alpha}^{\operatorname{div}}(p) & =(N+2) \frac{\lambda^{2}}{18 N^{2}} S_{a}(p) u_{\alpha}+\left(\frac{\lambda^{2} v}{18 N^{2}}\right)^{2}\left[\left(T_{a}^{(0)} T_{a}^{(I)}+T_{a}^{(I, 2)}\right) \delta_{\alpha \gamma}+A_{\alpha \beta}\left(T_{a}^{(0)} D_{1}^{\beta \gamma}+D_{2}^{\beta \gamma}\right)\right] B_{\gamma} \\
& +\frac{\lambda^{2}}{18 N^{2}} A_{\alpha \beta}\left\{\left[\left(\left(T_{a}^{(0)}\right)^{2}+T_{a}^{(I)}\right) \delta_{\beta \rho}+\left(T_{a}^{(0)} D_{0}^{\beta \gamma}+\tilde{D}_{1}^{\beta \gamma}\right) A_{\gamma \rho}\right]\left(M_{\rho}^{2}-M^{0} u_{\rho}\right)+T_{a}^{(0)} T_{\beta, F}\right\}(.85)
\end{aligned}
$$

The divergent quantities $D_{i}^{\alpha \beta}$ with $i \in\{0,1,2\}$ and $\tilde{D}_{1}^{\alpha \beta}$ are obvious generalisations of those appearing in (32) and (38) with $\Gamma_{0}^{\alpha \beta}$ and $\Gamma_{1}^{\alpha \beta}$ given by

$$
\Gamma_{0}^{\alpha \beta}(p)=-\frac{i}{18 N^{2}} \int_{k} \Gamma_{\alpha \beta}(p, k) G_{a}^{2}(k), \quad \Gamma_{1}^{\alpha \beta}(p)=-\frac{i}{18 N^{2}} \int_{k} \Gamma_{\alpha \beta}(p, k) G_{a}^{2}(k) I_{a, F}(k) .
$$

These matrices inherit from $\Gamma_{\alpha \beta}$ some relations between its elements which are analogous to (81) and (83):

$$
\begin{array}{ll}
D_{i}^{\sigma \sigma}-D_{i}^{\pi \sigma}=D_{i}^{\pi \pi}-D_{i}^{\sigma \pi}, & \tilde{D}_{1}^{\sigma \sigma}-\tilde{D}_{1}^{\pi \sigma}=\tilde{D}_{1}^{\pi \pi}-\tilde{D}_{1}^{\sigma \pi}, \\
D_{i}^{\sigma \pi}=(N-1) D_{i}^{\pi \sigma}, & \tilde{D}_{1}^{\sigma \pi}=(N-1) \tilde{D}_{1}^{\pi \sigma} .
\end{array}
$$

In order to determine the counterterms one has to use (84) and (85) in the renormalisation conditions for the two propagators (50), (51), supplemented with the terms given in (172), and in the renormalisation condition of the equation of state (65). In what follows we review, without giving the lengthy explicit expressions, how one can obtain the counterterms and the consistency conditions similar to (44). 
The vanishing of the coefficients of $v^{0}, v^{2}, T_{\sigma, F}$, and $T_{\pi, F}$ in the two renormalisation conditions for $G_{\pi}(p), G_{\sigma}(p)$ results in eight conditions for the determination of five counterterms: $\delta m_{2}^{2}, \delta \lambda_{0}^{A}, \delta \lambda_{0}^{B}$, $\delta \lambda_{2}^{A}$, and $\delta \lambda_{2}^{B}$. Out of them $\delta \lambda_{2}^{A}$ and $\delta \lambda_{2}^{B}$ are obtained from the vanishing of the coefficients of $v^{2}$. The vanishing of the divergent coefficients of $T_{\sigma, F}$ and $T_{\pi, F}$ in any one of the propagators determines $\delta \lambda_{0}^{A}$ and $\delta \lambda_{0}^{B}$. The condition to obtain the same expressions from the renormalisation conditions of the other propagator reduces to the equation

$$
\begin{array}{r}
\lambda^{2}(N+2)\left[C_{1} T_{a}^{(0)}-C_{0} T_{a}^{(I)}-3(N+2)\left(C_{0} D_{1}^{\pi \pi}-C_{1} D_{0}^{\pi \pi}+D_{1}^{\sigma \pi} D_{0}^{\pi \sigma}-D_{0}^{\sigma \pi} D_{1}^{\pi \sigma}\right)\right] \\
+6 N\left(\lambda C_{1}+N C_{0}\right)=0
\end{array}
$$

where $C_{i}=(N-1) D_{i}^{\pi \sigma}-D_{i}^{\sigma \pi}$. Using (87) this consistency condition is identically satisfied. The same way, in order to obtain the same expression for $\delta m_{2}^{2}$ from the vanishing of the coefficients of $v^{0}$ in either one of the two renormalisation conditions for the propagators one needs

$$
\begin{aligned}
{\left[\lambda^{2}(N+6) T_{a}^{(0)}\right.} & \left.+6 N\left(\lambda+\delta \lambda_{0}^{B}\right)\right]\left(D_{0}^{\sigma \sigma}+D_{0}^{\sigma \pi}-D_{0}^{\pi \sigma}-D_{0}^{\pi \pi}\right) \\
& +\lambda^{2}(N+6)\left(\tilde{D}_{1}^{\sigma \sigma}+\tilde{D}_{1}^{\sigma \pi}-\tilde{D}_{1}^{\pi \sigma}-\tilde{D}_{1}^{\pi \pi}\right)=0
\end{aligned}
$$

By (86), this is again identically satisfied. As a partial summary we emphasise that the eight conditions renormalising the propagator equations of the $O(N)$ model in the broken phase are algebraically satisfied with the five counterterms, independent of the form of the asymptotic propagator $G_{a}$.

Turning now to the equation of state, one can subtract its renormalisation condition from the renormalisation condition of $G_{\sigma}$, and require the cancellation of the term proportional to $T_{\sigma, F}, T_{\pi, F}$, $v^{0}$ and $v^{2}$. We can easily find the following relations:

$$
\delta \lambda_{2}^{A}=\delta \lambda_{0}^{A}, \quad \delta \lambda_{2}^{B}=\delta \lambda_{0}^{B}, \quad \delta m_{0}^{2}=\delta m_{2}^{2}, \quad \delta \lambda_{4}=\delta \lambda_{2}^{A}+2 \delta \lambda_{2}^{B}+\frac{\lambda^{2}}{3 N}(N+8) T_{a}^{(0)} .
$$

The first two conditions above impose two consistency conditions on the coupling counterterms if one uses their expression as obtained from the renormalisation conditions for the propagators. These are complicated expressions, similar to that in (44), which now depend also on $N$ and relate several cut-off dependent integrals. As in the one-component case, these relations can be checked only numerically.

\section{Conclusions}

In this paper we have extended the renormalisation procedure developed in Ref. 24] to the case of momentum-dependent self-energy appearing when one goes beyond the Hartree-Fock level of truncation of the 2PI effective action. Two types of momentum-dependent truncations of scalar $\Phi^{4}$ type models were investigated. In the first case the asymptotic behaviour of the exact propagator remains unchanged relative to the tree-level, while in the second case the asymptotic behaviour of the exact propagator changes.

Working in the broken symmetry phase we introduced infrared safe auxiliary propagators having the right asymptotic behaviour in order to explore the divergence structure of the model. Beyond the overall divergences proportional to the zeroth and second power of the background field we found only such subdivergences which were proportional to the finite part of the tadpole integrals. This 
is the reason that no counterterms are needed beyond those appearing at the Hartree truncation of the 2PI effective potential. Requiring the vanishing of the coefficients of independent divergences, we gave explicit expressions for the counter couplings of the model in terms of some integrals of the auxiliary propagator.

For the first type of truncation investigated, our method reproduces the expressions of the counter couplings which were previously reported in the literature for the case of a real selfinteracting scalar field, but with no reference to any Bethe-Salpeter equation. Although, the divergence structure was known in the literature for the second type of truncation, the explicit expressions for the counter couplings, which are needed to make the equations of the theory finite, appear to our best knowledge for the first time in this paper. These expressions depend in a non-trivial way on the solution of a Bethe-Salpeter-like equation and can be determined only numerically. This was beyond the scope of our present study in which we concentrated mainly on the structural aspects of the renormalisation.

The proposed method treats the conditions for overall and subdivergence cancellation on equal footing in the propagator equation(s) and the equation of state. Therefore, the number of conditions is larger than the actual number of counterterms. As shown in this paper, these conditions are algebraically fulfilled for the first type of truncation, both in the case of a one-component and a multi-component scalar field $(\mathrm{O}(\mathrm{N})$ model $)$. For the second type of truncation at least some part of the consistency conditions can be verified only numerically. It is quite impressive, though, that in this case the consistency of the propagator renormalisation can be proved algebraically. In the explicit demonstration the internal structure of the Bethe-Salpeter-like matrix equation plays an essential role. It is the compatibility of the renormalised propagator equations with the renormalised equation of state which leads to those consistency equations which can be verified only numerically.

The auxiliary propagators applied for the separation of divergences introduce also the renormalisation scale $M_{0}$. The explicit dependence of the counter couplings on this scale allows to establish the renormalised trajectory of the 2PI approximation in a higher-dimensional coupling space corresponding to the larger number of independent bare couplings necessary for its consistent renormalisation than in the perturbative case. Even when one restricts the beta-functions to leading (e.g. $\mathcal{O}\left(\lambda^{2}\right)$ ) order one finds the perturbative beta-function only for the coefficient of the $v^{4}$ term in the 2PIfunctional. It is interesting to note that beyond the perturbatively higher order contributions to the counter couplings also the appearance of the asymptotic propagator $G_{a}(p)$ in the relevant integrals produces deviations from the perturbative behaviour of the beta-functions determined in the $2 \mathrm{PI}$ approximation.

When passing to the numerical investigations the spirit of our paper suggests to make use of the explicit expressions for the cut-off dependent counterterms in the cut-off regulated equations (for instance (9) and (13)) and verify numerically the cut-off independence of their solution. Alternatively, one can prescribe values for a number of physical quantities in terms of the renormalised one- and two-point functions. One attempts then the determination of the asymptotic behaviour of the propagator from a finite twice-subtracted dispersive representation satisfying the renormalisation prescriptions. Before proceeding to the solution of the self-consistent equations in the non-asymptotic regime one has to check the consistency relations between asymptotic integrals. Numerical investigation of these questions essential for the validity of the renormalisation methods proposed for the 2PI approximation, will be reported elsewhere. 


\section{A Appendix A: Divergence structures for the two-loop truncation of the 2PI approximation}

The divergences of the integrals defined in (6), (17) and (8) can be most conveniently explored using Pauli-Villars type terms obtained by adding and subtracting in the integrands the auxiliary propagator

$$
i G_{P V}^{-1}(p)=p^{2}-M_{0}^{2} .
$$

The simplest is to separate the logarithmic divergent part of the bubble integral from its finite part. They read as

$$
I_{\mathrm{div}}=-\left.i \int_{k} G_{P V}^{2}(k)\right|_{\mathrm{div}}=: T_{d}^{(0)}, \quad I_{F}(p)=-i \int_{k}\left[G(k) G(k+p)-G_{P V}^{2}(k)\right],
$$

where the divergent piece is clearly independent of the external momentum.

For the propagator appearing in (10) one uses the identity

$$
G(p)=G_{P V}(p)-i G(p) G_{P V}(p)\left(M^{2}-M_{0}^{2}+\frac{\lambda^{2}}{2} v^{2} I_{F}(p)\right) .
$$

Solving (93) for $G(p)$ and expanding it around $G_{P V}(p)$ this equality can be rearranged also as

$$
G(p)=G_{P V}(p)+\delta G(p), \quad \delta G(p)=-i G_{P V}^{2}(p)\left(M^{2}-M_{0}^{2}+\frac{\lambda^{2} v^{2}}{2} I_{F}(p)\right)+G_{r}(p),
$$

where $G_{r}(p)$ is the regular part of the propagator, which contains at least three powers of $G_{P V}(p)$. It behaves asymptotically as $\sim p^{-6}$ and produces a convergent contribution when integrated over momentum.

With help of the representation (94) the divergences and the finite part of the tadpole integral are easily separable. After replacing $I_{F}(p)$ by $I_{P V, F}(p)$ in (94) (since the contribution of the difference to the tadpole is finite) the following partitioning is found:

$$
T[G]=T\left[G_{P V}\right]+\left(M^{2}-M_{0}^{2}\right) T_{d}^{(0)}-\frac{1}{2} \lambda^{2} v^{2} i \int_{p} G_{P V}^{2}(p) I_{P V, F}(p)+T_{F}[G] .
$$

Here one introduces the notations

$$
T\left[G_{P V}\right]=\left.\int_{p} G_{P V}(p)\right|_{\mathrm{div}}=: T_{d}^{(2)}, \quad T_{d}^{(I)}:=-\left.i \int_{p} G_{P V}^{2}(p) I_{P V, F}(p)\right|_{\mathrm{div}},
$$

and obtains the first entry in (15).

The setting-sun integral is rewritten after the replacement $G(p)=G_{P V}(p)+\delta G(p)$ is made for each of the three propagators as

$$
S(p=0, G)=S_{P V}(0)+3 \int_{k} \delta G(k) I_{P V}(k)+S_{F}^{(1)},
$$

where $S_{P V}(0)=-\left.i \int_{k} \int_{q} G_{P V}(k) G_{P V}(q) G_{P V}(k+q)\right|_{\text {div }}$. In the integral above one decomposes $I_{P V}(k)$ into a divergent and a finite part. For the piece proportional to the divergent part $I_{P V \text {,div }}=T_{d}^{(0)}$ one 
uses $\delta G(k)=G(p)-G_{P V}(k)$, while when taking the finite part of the bubble one substitutes $\delta G(k)$ from (94). With help of (96) the following representation for the divergent part is obtained:

$$
S_{\text {div }}(0, G)=S_{P V}(0)+3\left(T[G]-T_{d}^{(2)}\right) T_{d}^{(0)}+3\left(M^{2}-M_{0}^{2}\right) T_{d}^{(I)}-\left.\frac{3}{2} \lambda^{2} v^{2} i \int_{k} G_{P V}^{2}(k) I_{F}(k) I_{P V, F}(k)\right|_{\text {div }} .
$$

The divergent part of the integral above can be obtained by replacing $I_{F}(k)$ by $I_{P V, F}(k)$ (the difference gives a finite contribution). Then, introducing another cut-off dependent integral

$$
T_{d}^{(I, 2)}:=-\left.i \int_{k} G_{P V}^{2}(k) I_{P V, F}^{2}(k)\right|_{\mathrm{div}},
$$

one obtains the last entry in (15).

\section{B Divergence structures for the truncation of the 2PI ap- proximation at $\mathcal{O}\left(\lambda^{2}\right)$ skeleton diagram level}

The analysis goes quite in parallel with that given in Appendix A. One defines the basic divergent integrals to be used in the exploration of the divergence structure:

$$
\begin{aligned}
T_{a}^{(2)} & :=\left.\int_{p} G_{a}(p)\right|_{\text {div }}, & T_{a}^{(0)} & :=-\left.i \int_{p} G_{a}^{2}(p)\right|_{\text {div }}{ }^{\prime} \\
T_{a}^{(I)} & :=-\left.i \int_{p} G_{a}^{2}(p) I_{a, F}(p)\right|_{\text {div }}, & T_{a}^{(I, 2)} & :=-\left.i \int_{p} G_{a}^{2}(p) I_{a, F}^{2}(p)\right|_{\text {div }} .
\end{aligned}
$$

Making use of (17), (19) and (21), the identity corresponding to (93) reads

$$
G(p)=G_{a}(p)-i G(p) G_{a}(p)\left[M^{2}-M_{0}^{2}+\Pi_{0}(p)+\Pi_{2,0}(p)+\Pi_{r}(p)\right] .
$$

Solving (101) for $G(p)$ and expanding around $G_{a}(p)$ one obtains

$$
G(p)=G_{a}(p)+\delta G(p), \quad \delta G(p):=-i G_{a}^{2}(p)\left[M^{2}-M_{0}^{2}+\frac{1}{2} \lambda^{2} v^{2} I_{F}(p)+\Pi_{2,0}(p)\right]+G_{r}(p),
$$

where $G_{r}(p)$ contains also the contribution of $\Pi_{r}(p)$. Power counting shows that for asymptotically large values of $p$ one has $G_{r}(p) \sim p^{-6}$.

Eq. (102) is used to separate the divergence of the tadpole integral (see (24)), also to analyse the divergence structure of the momentum-dependent setting-sun integral and, eventually to obtain the integral representation of $S_{0, F}(p)\left(\right.$ e.g. $\left.\Pi_{2,0}(p)\right)$.

\section{Acknowledgements}

Work supported by the Hungarian Scientific Research Fund (OTKA) under Contract Nos. T046129 and T068108. Zs. Sz. is supported by OTKA Postdoctoral Grant no. PD 050015. 


\section{References}

[1] J. C. Collins, Renormalisation, Cambridge University Press, Cambridge, England, 1984.

[2] H. van Hees and J. Knoll, Phys. Rev. D 65 (2002) 025010 hep-ph/0107200].

[3] H. van Hees and J. Knoll, Phys. Rev. D 65 (2002) 105005 hep-ph/0111193.

[4] H. van Hees and J. Knoll, Phys. Rev. D 66 (2002) 025028 hep-ph/0203008.

[5] J.-P. Blaizot, E. Iancu and U. Reinosa, Phys. Lett. B568 (2003) 160 hep-ph/0301201.

[6] J.-P. Blaizot, E. Iancu and U. Reinosa, Nucl. Phys. A736 (2004) 149 hep-ph/0312085].

[7] F. Cooper, B. Mihaila, J. F. Dawson, Phys. Rev. D 70 (2004) 105008 hep-ph/0407119.

[8] F. Cooper, J. F. Dawson, B. Mihaila, Phys. Rev. D 71 (2005) 096003 hep-ph/0502040].

[9] E. Calzetta and B. L. Hu, Phys. Rev. D 35 (1987) 495.

[10] A. Arrizabalaga and J. Smit, Phys. Rev. D 66 (2002) 065014 hep-ph/0207044.

[11] M.E. Carrington, G. Kunstatter, H. Zaraket, Eur. Phys. J. C 42 (2005) 253 hep-ph/0309084.

[12] J. Berges, Sz. Borsányi, U. Reinosa and J. Serreau, Ann. Phys. 320 (2005) 344 [hep-ph/0503240].

[13] A. Arrizabalaga, J. Smit and A. Tranberg, Phys. Rev. D 72 (2005) 025014 hep-ph/0503287.

[14] A. Arrizabalaga and U. Reinosa, Nucl. Phys. A785 (2007) 234 hep-ph/0609053.

[15] J. Berges, Sz. Borsányi, U. Reinosa and J. Serreau, Phys. Rev. D 71 (2005) 105004 hep-ph/0409123.

[16] U. Reinosa and J. Serreau, JHEP 0607 (2006) 028 hep-th/0605023.

[17] Sz. Borsányi and U. Reinosa, Phys. Lett. B661 (2008) 88 arXiv:0709.2316].

[18] U. Reinosa and J. Serreau, JHEP 0711 (2007) 097 arXiv:0708.0971.

[19] H. Verschelde, Phys. Lett. B497 (2001) 165 hep-th/0009123.

[20] G. Smet, T. Vanzielighem, K. Van Acoleyen, H. Verschelde, Phys. Rev. D 65 (2002) 045015 hep-th/0108163.

[21] J. Baacke and A. Heinen, Phys. Rev. D 67 (2003) 105020 hep-ph/0212312.

[22] J. Baacke and S. Michalski, Phys. Rev. D 70 (2004) 085002 hep-ph/0407152].

[23] A. Jakovác Phys. Rev. D 76 (2007) 125004 hep-ph/0612268.

[24] G. Fejős, A. Patkós and Zs. Szép, Nucl. Phys. A803 (2008) 135 [arXiv:0711.2933].

[25] S. Weinberg, Phys. Rev. 118 (1959) 838.

[26] Yu. B. Ivanov, F. Riek, J. Knoll, Phys. Rev. D 71 (2005) 105016 hep-ph/0502146. 